\title{
Integrated Stiffness Analysis of Redundant Parallel Manipulator Based on Finite Element Method
}

\author{
Songtao Wang ${ }^{\mathrm{a}}$, Gang Cheng ${ }^{\mathrm{a}, *}$, Yusong Pang ${ }^{\mathrm{b}}$, Gabriel Lodewijks ${ }^{\mathrm{b}}$

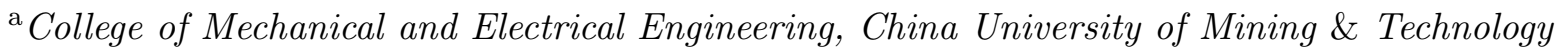 \\ Xuzhou 221116, China \\ ${ }^{\mathrm{b}}$ Faculty Mechanical, Maritime and Materials Engineering, Delft University of Technology \\ Delft, 2628 VX, The Netherlands
}

\begin{abstract}
An integrated stiffness model is established for a Planar Parallel Manipulator (PPM) with actuation redundancy based on Finite Element Method (FEM), and the static stiffness, dynamitic stiffness and moving stiffness of the PPM are analyzed according to the integrated stiffness model. Firstly, a dynamic model of flexible plane beam element is created as a basic unit for branches. Secondly, each branch is assembled in generalized coordinates, and the integrated stiffness model of the PPM is established. Then calculation and simulation for the static stiffness, dynamitic stiffness and moving stiffness are carried out. The results show that the static stiffness and dynamitic stiffness are related with the position and posture of the PPM. The moving stiffness shows that the elastic deformations cause the oscillation of the PPM. In this paper, three stiffness models are unified in the integrated stiffness model, which improves the efficiency of the stiffness calculation and mechanism design.
\end{abstract}

Keywords: Parallel Manipulator; Finite Element Method; Integrated Stiffness; Dynamics

\section{Introduction}

Parallel manipulators have many advantages including high load capacity, high accuracy and compact structure. Parallel manipulators are usually used in high loading and high speed working conditions [1], so the stiffness is an important indicator of the parallel mechanism performance. The stiffness analysis of parallel manipulators includes three aspects: static stiffness analysis, dynamic stiffness analysis and moving stiffness analysis.

Many scholars have studied the stiffness of parallel manipulators. The Jacobian matrix-based method is presented by Gosselin and other scholars $[2,3,4]$. The Jacobian matrix is used to calculate a stiffness matrix and the analysis is carried out for the entire workspace. The results show that the stiffness is depended on the workspace. A similar methodology is described in reference [5], and the links are considered to be flexible. Several authors use matrix product method

\footnotetext{
${ }^{*}$ Corresponding author.

Email address: chg@cumt.edu.cn (Gang Cheng).
} 
to analyze the stiffness [6]. Ceccarelli and Carbone use three set of matrices to get the stiffness matrix [7]. The same procedure is described and is completed with lumped parameters [8]. Finite element calculating method is another way to calculate the stiffness. This method constructs the stiffness matrix by finite element formulation. Structural matrices of the elements are built and assembled including joint stiffness $[9,10]$. Analytical-experimental method is an important method to analyze stiffness, which obtains experimental results in analytical stiffness matrix calculation procedures [11]. Finite elements and experimental results are combined to calculate the static stiffness of a 3T1R manipulator $[12,13,14]$, and authors use dial indicators to measure experimental Cartesian displacements under an applied external load and get the stiffness of the parallel manipulators. Above studies focused on static stiffness analysis and achieve good applications in practice. However, the above studies do not consider the dynamics of parallel manipulators in modeling result that they are not able to analyze dynamic stiffness and moving stiffness. The dynamic stiffness analysis and moving stiffness analysis of parallel manipulators have been studied $[15,16]$. Above researches successfully analyze the different stiffness, but the methods are not unified, which increases the research difficulty in analyzing different stiffness. In this paper, considering above researches, a Finite Element Method (FEM) is used to establish integrated stiffness model for parallel manipulators based on the finite element theory and the Lagrange dynamic equation, and the integrated stiffness model of parallel manipulators can be used to analyze static stiffness, dynamic stiffness and moving stiffness at the same time.

This paper is organized as follows: In Section 1, the system structure of the planar parallel manipulator is introduced. In Section 2, the integrated stiffness model of the planar parallel manipulator is established according to the finite element method. In Section 3, numerical calculation and simulation are carried out. Finally this paper is summarized.

\section{System Structure}

The Planar Parallel Manipulator (PPM) with actuation redundancy is a 3-RR planar parallel manipulator. "3" represents that the PPM has three branches. "R" represents the revolute joint and "RR" represents that each branch has two revolute joints. The physical structure of the PPM is shown in Fig. 1. The PPM uses three PW DC motors as the drivers to drive the active arms. The movement of actuator is determined by the motions of three passive arms. The topology structure of the PPM is shown in Fig. 2. $A_{i}\left(x_{a i}, y_{a i}\right)$ and $B_{i}\left(x_{b i}, y_{b i}\right)$ represent the joint positions. $P$ represents the position of the actuator with a coordinate $P(x, y)$ and $\theta_{a i}$ represents the angle between $A_{i} B_{i}$ and $x$ axis. $\theta_{b i}$ represents the angle between $B_{i} P$ and $x$ axis. In the above, $i=1$, 2,3 . The length of active arm and passive arm is equal, which is denoted as $L$.

\section{$3 \quad$ Finite Element Analysis}

\subsection{Discrete Structure and Analysis Element}

Assume that $A_{i} B_{i}$ and $B_{i} P$ are the deformable links in the PPM, and the joint deformation can be neglected (The system cumulative error caused by joint deformation is smaller because the branches in parallel manipulator are generally shorter, and another reason is that parallel manipulator is almost a closed loop system having the certain restraining action for the joint 


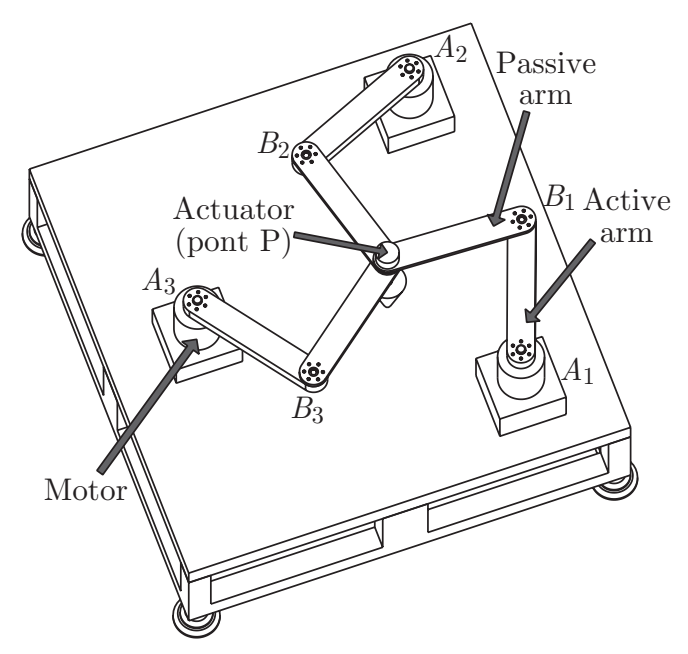

Fig. 1: Physical structure of the PPM with 2$\mathrm{DOF}$

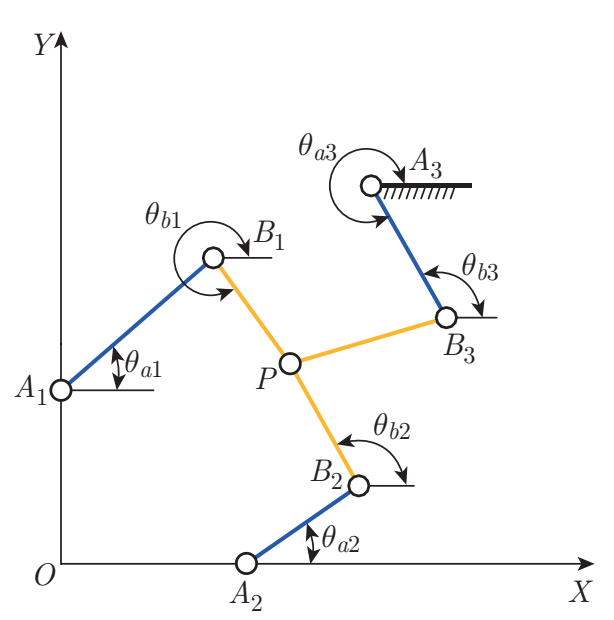

Fig. 2: Topology structure of the PPM with 2DOF

deformation). $A_{i} B_{i}$ and $B_{i} P$ are the deformable links each of which can be assumed as one unit, so there are six deformable units in the PPM.

According to the structural characteristics of the PPM, the plane beam element with a rectangular section is chosen as the basic unit, as shown in Fig. 3. $A$ and $B$ represent the nodes of the plane beam element. The node displacements can be expressed in local coordinate system $A-X Y Z$. $q_{1}$ and $q_{4}$ represent the elastic displacements along $X$ axis of nodes $A$ and $B$, respectively. $q_{2}$ and $q_{5}$ represent the elastic displacements along $Y$ axis of nodes $A$ and $B$, respectively. $q_{3}$ and $q_{6}$ represent the bending angles around $Z$ axis of nodes $A$ and $B$, respectively. Each local coordinate of elements is not unified, so it is not convenient to study the mechanical structure. Considering the reason mentioned in previous, the unification generalized coordinates is established as shown in Fig. 2.

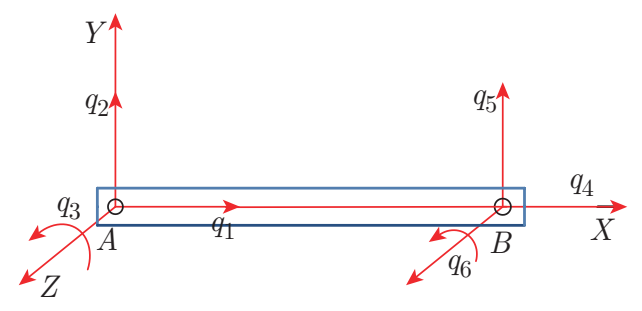

Fig. 3: Plane beam element

\subsection{Unit Displacement Interpolation Function}

Assume that the plane beam element occurs axial and lateral bending deformation, and $q=\left[\begin{array}{ll}q_{1} & q_{2}\end{array}\right.$ $\left.q_{3} \quad q_{4} q_{5} q_{6}\right]^{T}$ represents the generalized coordinate vector of plane beam element. In the unit coordinates (local coordinates), the elastic displacement along $X, Y$ direction and the elastic angle displacement around $Z$ axis can be expressed as the functions of $q$. The functions of the elastic displacement along $X, Y$ axis and the elastic angle displacement around $Z$ axis are denoted as $W_{x}(x, t), W_{y}(x, t)$ and $\theta_{z}(x, t)$, respectively. According to the mechanism characteristics and accuracy requirement, the elastic displacements along $X, Y$ axis use a linear differential function, 
and the elastic angle displacement uses a three cubic interpolation function. According to the boundary conditions, the following formula can be derived:

$$
\begin{aligned}
W_{x}(x, t) & =N_{A}^{T} q \\
W_{y}(x, t) & =N_{B}^{T} q \\
\theta_{z}(x, t) & =N_{c}^{T} q
\end{aligned}
$$

where $q$ is the generalized coordinate vector. $N_{A}, N_{B}$ and $N_{C}$ are the interpolation vectors, and each of them is the function of $x$.

During movement, the coupling effect between the rigid motion and the elastic motion of the unit can be ignored because the elastic motion of the unit is smaller. So the absolute velocity at any point in a unit can be considered as the superposition by its rigid velocity and elastic velocity, and the absolute acceleration at any point in a unit is the superposition by its rigid acceleration and elastic acceleration. The velocity for any point in a unit can be expressed as follows:

$$
\begin{aligned}
& \dot{W}_{a x}(x, t)=\dot{W}_{r x}(x, t)+\dot{W}_{x}(x, t) \\
& \dot{W}_{a y}(x, t)=\dot{W}_{r y}(x, t)+\dot{W}_{y}(x, t)
\end{aligned}
$$

where $\dot{W}_{a x}(x, t), \dot{W}_{a y}(x, t), \dot{W}_{r x}(x, t), \dot{W}_{r y}(x, t), \dot{W}_{x}(x, t)$ and $\dot{W}_{y}(x, t)$ represent the absolute velocities, the rigid velocities and the elastic velocities along $X$ and $Y$ axis, respectively.

\subsection{Finite Element Analysis of the Unit}

Assume that the unit mass in each section is concentrated in the center axis and the influence of the revolute energy can be ignored. Then the kinetic energy of the unit can be expressed as follows:

$$
T=\frac{1}{2} \int_{0}^{L} m(x)\left[\left(\frac{d W_{a x}(x, t)}{d t}\right)^{2}+\left(\frac{d W_{a y}(x, t)}{d t}\right)^{2}\right] d x
$$

where $L, \rho$ and $A$ represent the unit length, the unit mass density and the unit cross-sectional area, respectively. $m(x)$ represents the function of unit mass distribution. For the beam element with uniform section, there is a function: $m(x)=\rho A$. Incorporating Eq. (4), Eq. (5) and Eq. (6), the following equation can be derived as:

$$
T=\frac{1}{2}\left(\dot{q}_{r}+\dot{q}\right)^{T} M_{e}\left(\dot{q}_{r}+\dot{q}\right)
$$

where $\dot{q}_{r}$ and $\dot{q}$ represent the rigid velocity and elastic velocity of the unit, respectively, and $M_{e}$ represents the unit mass matrix.

All deformation energy of the unit can be expressed as:

$$
V=\frac{1}{2} E \int_{0}^{l}\left[A\left(\frac{\partial W_{x}(x, t)}{\partial x}\right)^{2}+I_{z}\left(\frac{\partial^{2} W_{y}(x, t)}{\partial x^{2}}\right)^{2}\right] d x
$$

where $E$ is the tension-compression modulus of elasticity, and $I_{Z}$ is the main moment of inertia of beam element cross section to $Z$ axis. 
Taking Eq. (4) and Eq. (5) into Eq. (8), it can be gotten that:

$$
V=\frac{1}{2} q^{T} K_{e} q
$$

where $K_{e}$ is the unit stiffness matrix and $q$ is the velocity vector.

Taking Eq. (8) and Eq. (9) into Lagrange Equation: $\frac{d}{d t}\left(\frac{\partial T}{\partial \dot{q}}\right)-\frac{\partial T}{\partial q}+\frac{\partial V}{\partial q}=F$, and the dynamic equation of unit is obtained as:

$$
M_{e} \ddot{q}+K_{e} q=F_{e}+P_{e}+Q_{e}
$$

where $F_{e}$ and $P_{e}$ represent the external force vector and the inner force vector, respectively, and $Q_{e}$ represents the inertia force vector.

\subsection{Finite Element Analysis of Branch}

The active units $A_{i} B_{i}(i=1,2,3)$ are assumed as the cantilever beams. Considering the boundary conditions, the elastic angle displacements at node $B_{i}(i=1,2,3)$ are zeros. Nodes $B_{i}$ owe different angles because they belong to different components (unit $A_{i} B_{i}$ and unit $B_{i} P$ ). Due to nodes $B_{i}$ represent the revolute joints, the two curvatures around the axis of $B_{i}$ are zeros. Node $P$ is the point where the ends of three branched chains are intersected and $P$ represents the revolute joint. So the curvature at point $P$ is zero. Therefore, the number of non-zero local coordinates of $A_{i} B_{i}$ is three and the number of non-zero local coordinates of $B_{i} P$ is four, as shown in Fig. 4. According to the FEM, the node deformation of branch $A_{i} B_{i} P$ can be described by five generalized coordinates $U_{i}=\left[\begin{array}{lllll}u_{i 1} & u_{i 2} & u_{i 3} & u_{i 4} & u_{i 5}\end{array}\right]$, as shown in Fig. 5.

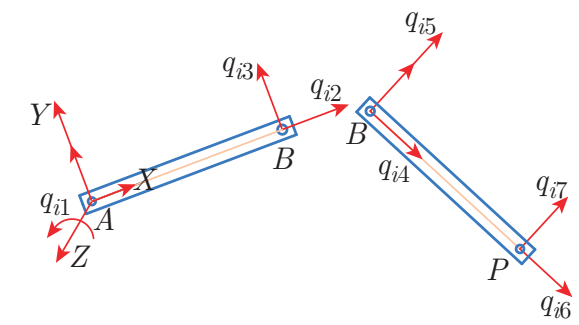

Fig. 4: Finite element model in local coordinates

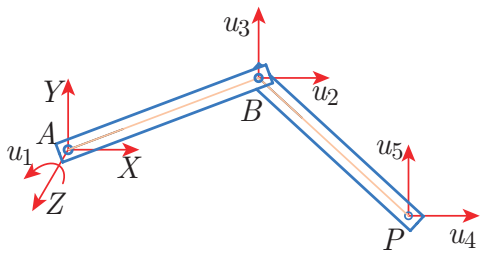

Fig. 5: Finite element model in generalized coordinates

According to the above analysis, $\theta_{i 1}$ is the angle between $A_{i} B_{i}$ and $X$-axis in chain $A_{i} B_{i} P$ and $\theta_{i 2}$ is the angle between $B_{i} P$ and $X$-axis, as shown in Fig. 1 . The transform matrix which transfers the $A_{i} B_{i}$ branch coordinates from the generalized coordinates $O-X Y Z$ to local coordinates $A_{i}$ $X Y Z$ is expressed as follows:

$$
R_{i 1}=\left[\begin{array}{ccc}
\cos \theta_{i 1} & \sin \theta_{i 1} & 0 \\
-\sin \theta_{i 1} & \cos \theta_{i 1} & 0 \\
0 & 0 & 1
\end{array}\right]
$$

Proceeding in the same way, the transform matrix which transfers the $B_{i} P$ branch coordinates 
from the generalized coordinates $O-X Y Z$ to local coordinates $B_{i}-X Y Z$ can be expressed as follows:

$$
R_{i 2}=\left[\begin{array}{ccc}
\cos \theta_{i 2} & \sin \theta_{i 2} & 0 \\
-\sin \theta_{i 2} & \cos \theta_{i 2} & 0 \\
0 & 0 & 1
\end{array}\right]
$$

Therefore, the relation between the local coordinates and the generalized coordinates for $A_{i} B_{i} P(i=$ $1,2,3)$ coordinates which can be expressed as follows:

$$
\left[\begin{array}{l}
q_{i 1} \\
q_{i 2} \\
q_{i 3} \\
q_{i 4} \\
q_{i 5} \\
q_{i 6} \\
q_{i 7}
\end{array}\right]\left[\begin{array}{ccc}
1 & 0 & 0 \\
0 & R_{i 1}^{*} & 0 \\
0 & R_{i 2}^{*} & 0 \\
0 & 0 & R_{i 2}^{*}
\end{array}\right]_{7 \times 5}\left[\begin{array}{l}
u_{i 1} \\
u_{i 2} \\
u_{i 3} \\
u_{i 4} \\
u_{i 5}
\end{array}\right]
$$

where: $R_{i 1}^{*}=\left[\begin{array}{cc}\cos \theta_{i 1} & \sin \theta_{i 1} \\ -\sin \theta_{i 1} & \cos \theta_{i 1}\end{array}\right], R_{i 2}^{*}=\left[\begin{array}{cc}\cos \theta_{i 2} & \sin \theta_{i 2} \\ -\sin \theta_{i 2} & \cos \theta_{i 2}\end{array}\right]$.

The compact matrix form of Eq. (13) is

$$
q^{i}=C_{i} U_{i}
$$

where: $q^{i}=\left[\begin{array}{lllllll}q_{i 1} & q_{i 2} & q_{i 3} & q_{i 4} & q_{i 5} & q_{i 6} & q_{i 7}\end{array}\right]^{T}, U_{i}=\left[\begin{array}{lllll}u_{i 1} & u_{i 2} & u_{i 3} & u_{i 4} & u_{i 5}\end{array}\right]^{T}, C_{i}=\left[\begin{array}{ccc}1 & 0 & 0 \\ 0 & R_{i 1}^{*} & 0 \\ 0 & R_{i 2}^{*} & 0 \\ 0 & 0 & R_{i 2}^{*}\end{array}\right]$.

The number of non-zero generalized coordinates of $A_{i} B_{i}(i=1,2,3)$ is three according to its boundary. By incorporating the dynamic equations from Eq. (10) to Eq. (14), the dynamic equation of $A_{i} B_{i}$ can be written as:

$$
M_{e}^{i 1} \ddot{q}^{i 1}+K_{e}^{i 1} q^{i 1}=F_{e}^{i 1}+P_{e}^{i 1}+Q_{e}^{i 1}
$$

where $q^{i 1}$ is the generalized coordinate of the unit for branch $A_{i} B_{i}$ and superscript " $i 1$ " repents the first unit in the $i$ branch. $M_{e}^{i 1}$ is the mass matrix of branch $A_{i} B_{i}$, and $K_{e}^{i 1}$ is the stiffness matrix of branch $A_{i} B_{i} . F_{e}^{i 1}$ is the external force vector which is applied to branch $A_{i} B_{i}$, and $P_{e}^{i 1}$ is the force vector which other units give to branch $A_{i} B_{i} . Q_{e}^{i 1}$ is the inertia force vector of branch $A_{i} B_{i}$.

The number of non-zero generalized coordinates of $B_{i} P(i=1,2,3)$ is four according to its boundary. By incorporating Eq. (10) to Eq. (14), the dynamic equation of branch $B_{i} P$ can easily be derived as:

$$
M_{e}^{i 2} \ddot{q}^{i 2}+K_{e}^{i 2} q^{i 2}=F_{e}^{i 2}+P_{e}^{i 2}+Q_{e}^{i 2}
$$


where $q^{i 2}$ is the generalized coordinates of the unit for branch $B_{i} P$ and superscript " $i 2$ " repents the second unit in the $i$ branch. $M_{e}^{i 2}$ is the mass matrix of branch $B_{i} P$, and $K_{e}^{i 2}$ is the stiffness matrix of branch $B_{i} P . F_{e}^{i 2}$ is the external force vector which is applied to branch $B_{i} P$, and $P_{e}^{i 2}$ is the force vector which other units give to branch $B_{i} P . Q_{e}^{i 2}$ is the inertia force vector of branch $B_{i} P$.

Taking Eq. (11) and Eq. (12) into Eq. (15) and Eq. (16), respectively, and the dynamic equations of $A_{i} B_{i}$ and $B_{i} P$ can be derived in the generalized coordinates. After the above analysis, the dynamic equation of branch $A_{i} B_{i} P$ can be derived as:

$$
M^{i} \ddot{U}_{i}+K^{i} U_{i}=F^{i}+P^{i}+Q^{i}(i=1,2,3)
$$

where: $U_{i}$ is the node coordinate of branch $A_{i} B_{i} P . M^{i}$ is the mass matrix of branch $A_{i} B_{i} P$, and $K^{i}$ is the stiffness matrix of branch $A_{i} B_{i} P . F^{i}$ is the external force vector which is applied to branch $A_{i} B_{i} P$, and $P_{e}^{i 1}$ is the force vector of branch $A_{i} B_{i} P_{i}$. $Q^{i}$ is the inertia force vector of branch $A_{i} B_{i} P$.

\subsection{Finite Element Analysis of the PPM}

According to the kinematics and dynamics constraints in the PPM, the dynamics of the PPM has been analyzed. The Eq. (18) shows the transform relationship between the generalized coordinates $U^{*}$ and the system coordinates $U$ as shown in Fig. 6 .

where: $U *=\left[\begin{array}{lllllllllllllll}u_{11} & u_{12} & u_{13} & u_{14} & u_{15} & u_{21} & u_{22} & u_{23} & u_{24} & u_{25} & u_{31} & u_{32} & u_{33} & u_{34} & u_{35}\end{array}\right]^{T}$,

$$
U=\left[\begin{array}{llllllllll}
u_{1} & u_{2} & u_{3} & u_{4} & u_{5} & u_{6} & u_{7} & u_{8} & u_{9} & u_{10}
\end{array}\right]^{T} .
$$

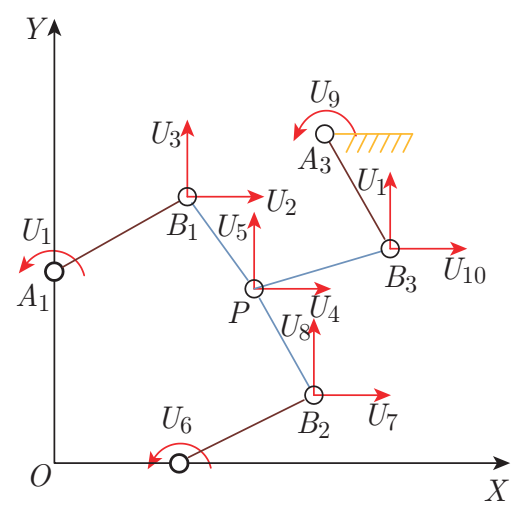

Fig. 6: Generalized coordinates of the system

In accordance with the constraint system equations, it can be gotten as:

$$
U^{*}=C U
$$

Substituting Eq. (18) into Eq. (17), the following equation can be obtained.

where: $M_{e}=\left[\begin{array}{ccc}M_{e}^{1} & & \\ & M_{e}^{2} & \\ & & M_{e}^{3}\end{array}\right], K_{e}=\left[\begin{array}{ccc}K_{e}^{1} & & \\ & K_{e}^{2} & \\ & & K_{e}^{3}\end{array}\right]$, 


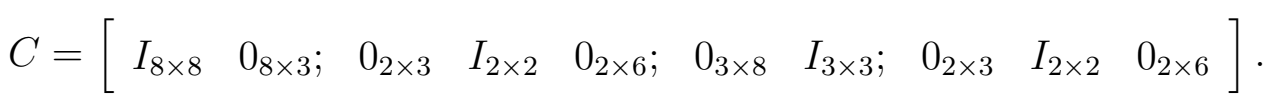

Substituting Eq. (18) into Eq. (19), the integrated stiffness model can be obtained.

$$
M \ddot{U}+K U=F
$$

where: $M=B^{T} \mathrm{MeB}, K=B^{T} \mathrm{KeB}$.

\subsection{Stiffness Analysis}

(1) Static stiffness analysis

Definition of the static stiffness is that the mechanical ability resists the deformation under static loading, so the static stiffness can be denoted as an evaluation index. The procedure for solving the static stiffness is shown in Fig. 7. The procedure is that the basic element is selected by analyzing the PPM, and the basic nodes and elements are numbered, and then the system stiffness matrix is made up from the stiffness matrixes of elements according to Eq. (20). Through analyzing system stiffness matrix, the static stiffness can be calculated out. By comparing the calculated static stiffness with the permissible stiffness it can be judged whether the PPM can meet the working requirements.

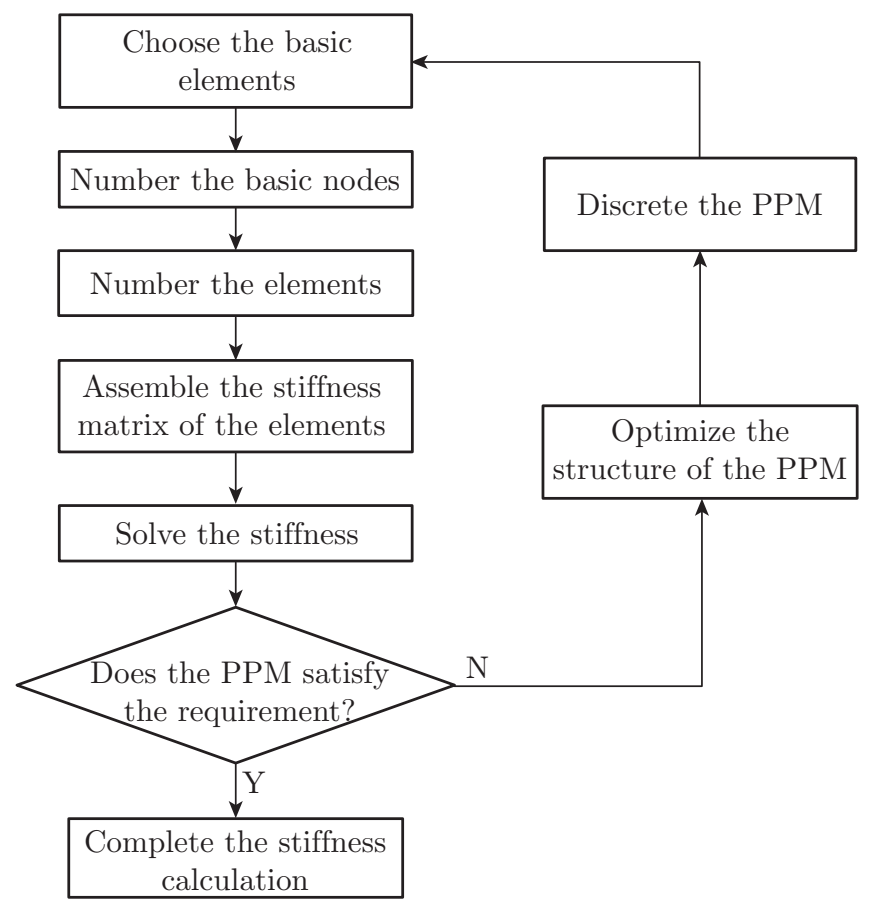

Fig. 7: Flowchart for solving the static stiffness

(2) Dynamic stiffness analysis

Definition of the dynamic stiffness is that the mechanical ability resists deformation under dynamic loading, and another definition is the dynamic force which caused unit amplitude. The evaluation index of the dynamic stiffness is inherent frequency. 
The dynamic stiffness equation of the PPM can be expressed as follow:

$$
\left.\begin{array}{l}
|D-\lambda I|=0 \\
D=K^{-1} M \\
\lambda=1 / \omega^{2}
\end{array}\right\}
$$

where $\omega$ is the inherent frequency vector and the minimum value is defined as $\omega_{0}$. By comparing $\omega_{0}$ with the permissible value, it can be judged whether the dynamic stiffness can meet the requirement.

(3) Moving stiffness analysis

The moving stiffness analysis usually represents the structure vibration when the PPM is working, and it has several evaluation indexes, such as elastic displacement, elastic velocity and elastic acceleration.

Applying the Runge-Kutta method to solve Eq. (20), the elastic displacement $U$ can be obtained. According to the derivative of $U$, the elastic velocity $\dot{U}$ and the elastic acceleration $\ddot{U}$ can be derived.

\section{Numerical Simulations}

In order to analyze the stiffness characteristics of the PPM, it is essential to set the structural parameters shown in Table 1 and the permissible stiffness values list in Table 2.

Table 1: Structural parameters of the PPM

\begin{tabular}{ccc}
\hline Structural parameter & Parameter value & Units \\
\hline Material & Aluminum & \\
Elastic modulus & 70 & $\mathrm{GPa}$ \\
Rod length $(L)$ & 0.244 & $\mathrm{~m}$ \\
Cross-sectional area & 0.004 & $\mathrm{~m}^{2}$ \\
Rotational inertia & 0.014 & $\mathrm{Kgm}^{2}$ \\
$A_{1}$ coordinates $\left(x_{a 1}, y_{a 1}\right)$ & $(0,0.25)$ & $\mathrm{m}$ \\
$A_{2}$ coordinates $\left(x_{a 2}, y_{a 2}\right)$ & $(0.43,0)$ & $\mathrm{m}$ \\
$A_{3}$ coordinates $\left(x_{a 3}, y_{a 3}\right)$ & $(0.43,0.5)$ & $\mathrm{m}$ \\
\hline
\end{tabular}

Table 2: Permissible stiffness values of the PPM

\begin{tabular}{ccc}
\hline Index type & Evaluation index & Value \\
\hline Static stiffness & Static stiffness value & $\geq 5 \times 10^{8}(\mathrm{Nm})$ \\
Dynamic stiffness & Inherent frequency & $\leq 1(\mathrm{~Hz})$ \\
Moving stiffness & Elastic displacement & $\leq 0.01(\mathrm{~mm})$ \\
\hline
\end{tabular}




\subsection{Static Stiffness Simulation}

The static stiffness values of the PPM are different when the actuator is in different positions. Therefore, it is necessary to study the static stiffness in working space. The minimum static stiffness is at the actuator by analyzing the characteristics of the PPM, so the static stiffness of the actuator can be used to judge whether the PPM can meet the stiffness requirement. The static stiffness of the actuator is not isotropic through qualitative analysis, so the static stiffness along $X$-axis and $Y$-axis are both calculated out in working space in order to obtain the static stiffness range. The calculated results are shown in Fig. 8.

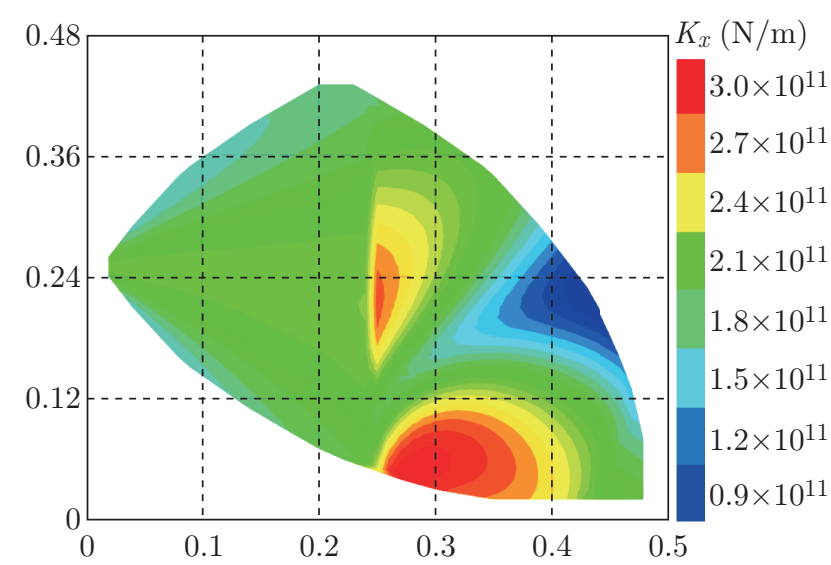

(a) Static stiffness along $X$-axis

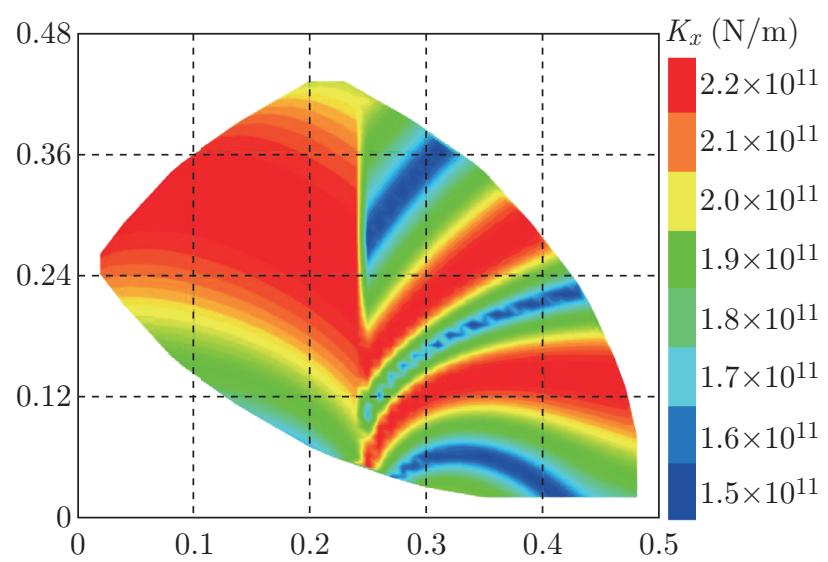

(b) Static stiffness along $Y$-axis

Fig. 8: Stiffness distribution of the PPM in the working space

If the PPM wants to operate normally, it requires that the minimum static stiffness in the working space is larger than the permissible stiffness. The simulation results show that the minimum static stiffness value along $X$-axis is $0.9 \times 10^{11} \mathrm{Nm}$ (as shown in Fig. 8 (a)) and the minimum static stiffness value along $Y$-axis is $1.5 \times 10^{11} \mathrm{Nm}$ (as shown in Fig. 8 (b)) which are both larger than the permissible value (as listed in Table 2), so the PPM satisfies the requirement of the static stiffness.

The FE software is used to simulate the static stiffness when the actuator is in the position $(0.25,0.29)$ in order to check the static stiffness calculated by FEM. The model is meshed and the constraints and loads are set in the FE software as shown in Fig. 9. Assuming that the PPM is subjected to a working resistance, and the working resistance along $X$-axis and $Y$-axis are both $1000 N$. The working resistance is set along $X$-axis as shown in Fig. 9 (a) and the force is set along $Y$-axis as shown in Fig. 9 (b).

The simulation results are shown in Fig. 10. Fig. 10 (a) shows the elastic displacement along $X$-axis $\left(\delta_{x}\right)$ and Fig. 10 (b) shows the elastic displacement along $Y$-axis $\left(\delta_{y}\right)$. According to the Fig. 10, the elastic displacement along $X$-axis at the actuator is $4.29 \times 10^{-7} \mathrm{~m}$ and the elastic displacement along $Y$-axis at the actuator is $3.57 \times 10^{-7} \mathrm{~m}$. According to the elastic displacement formula $K=F / \delta$, the static stiffness along $X$-axis and $Y$-axis can be calculated as $2.33 \times 10^{11}$ $\mathrm{N} / \mathrm{m}$ and $2.08 \times 10^{11} \mathrm{~N} / \mathrm{m}$, and the static stiffness at the same position calculated by FEM are $2.42 \times 10^{11} \mathrm{~N} / \mathrm{m}$ and $2.15 \times 10^{11} \mathrm{~N} / \mathrm{m}$. The two results calculated by FEM and FE software are at the same magnitude and the relative error is small. The results suggest that using FEM to calculate the static stiffness is feasible. At the same time the static stiffness calculated by FEM is larger than the static stiffness calculated by FE software, because the branches are divided into 


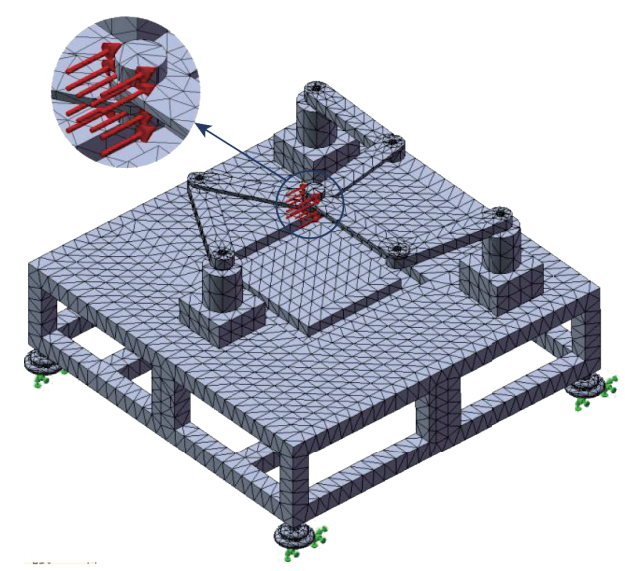

(a) Force along $X$-axis

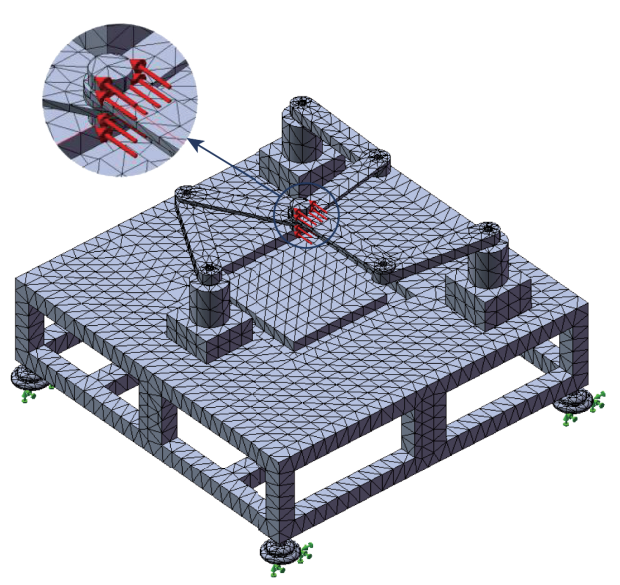

(b) Force along $Y$-axis

Fig. 9: Preprocessing in FE software

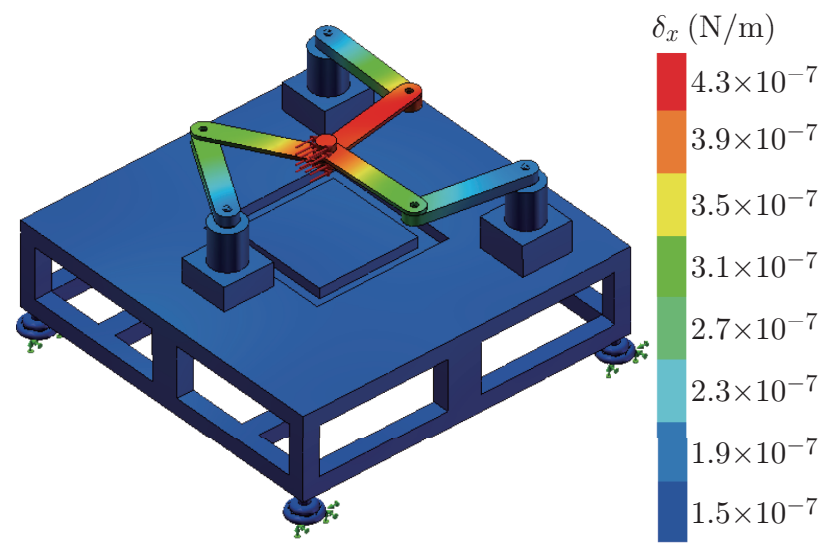

(a) Elastic displacement along $X$-axis

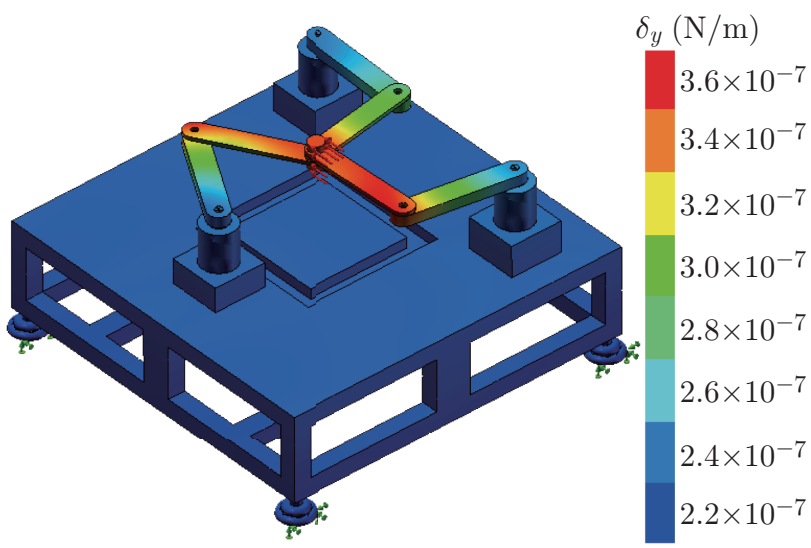

(b) Elastic displacement along $Y$-axis

Fig. 10: Elastic displacement

many meshes in the FE software.

\subsection{Dynamic Stiffness Simulation}

The inherent frequency of the PPM is also different when the manipulator is at different position, so the inherent frequency in the working space needs to be analyzed. By solving the stiffness model of the PPM, the inherent frequency $(\omega)$ of each position can be obtained. The inherent frequency distribution in the working space can be described in Fig. 11.

As seen in Fig. 11, the minimum inherent frequency is $1.7 \times 10^{6} \mathrm{~Hz}$ which is much larger than the permissible frequency listed in Table 2, so the PPM satisfies the demand of the dynamic stiffness.

\subsection{Moving Stiffness Simulation}

Moving stiffness analysis needs to consider the moving condition, so a movement trajectory must be assumed. The movement trajectory is set that the starting point is $(0.22,0.29)$ and the ending 


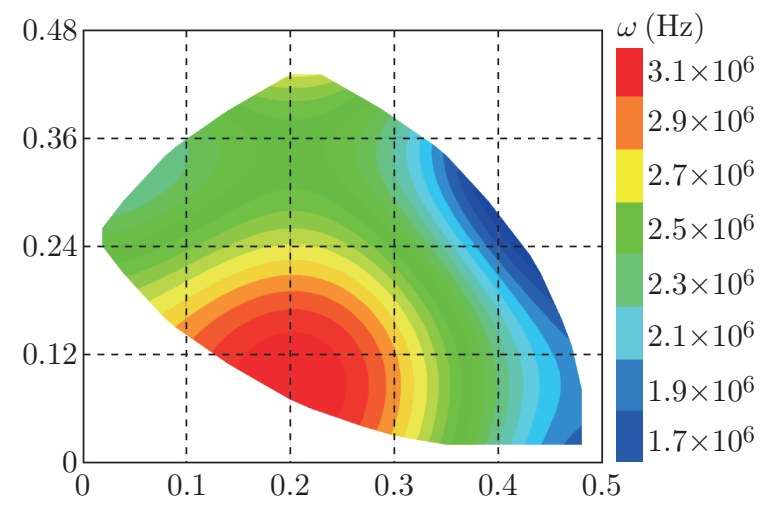

Fig. 11: Inherent frequency distribution of the PPM in the working space

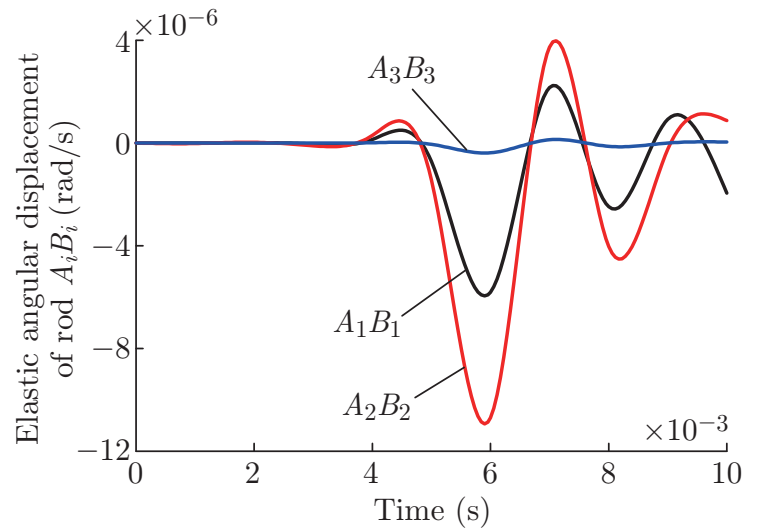

(a) Elastic angular displacement of link $A_{i} B_{i}$

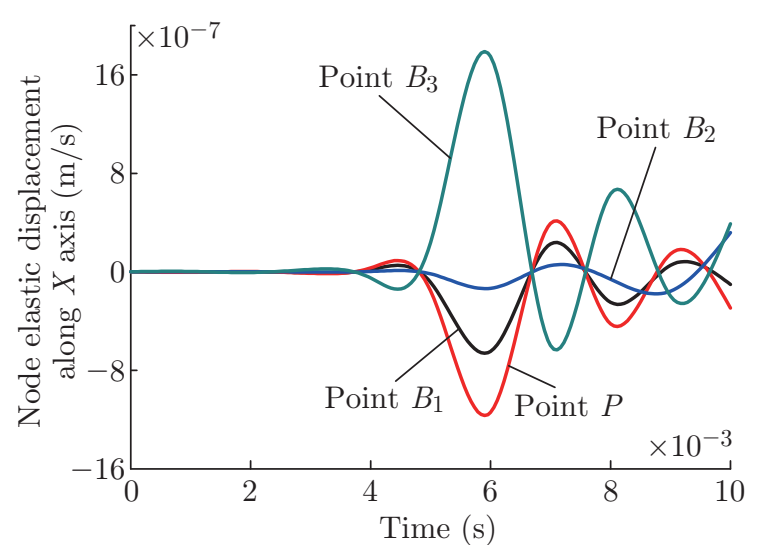

(b) Node elastic displacement along $X$ axis

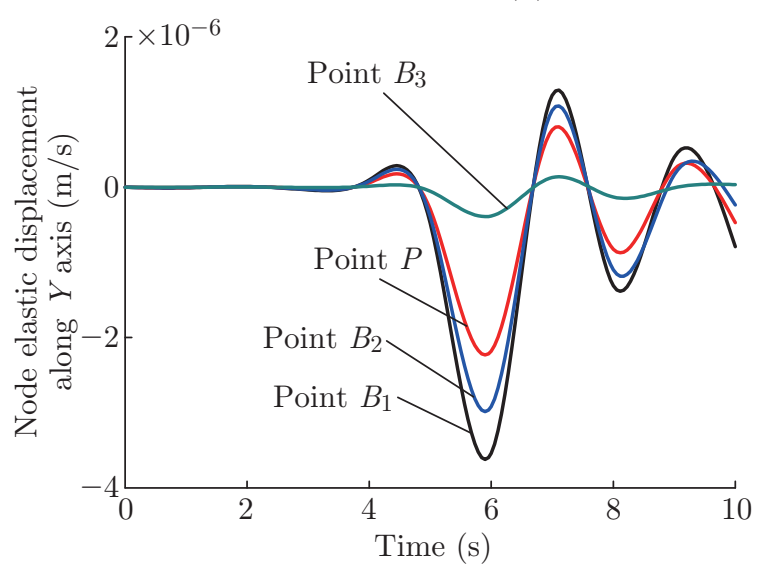

(c) Node elastic displacements along $Y$ axis

Fig. 12: Elastic displacements of the PPM

point is $(0.27,0.24)$, where the movement distance is $0.17 \mathrm{~m}$. The resistance force is $100 \sqrt{2} \mathrm{~N}$, and the speed is $5 \sqrt{2} \mathrm{~m} / \mathrm{s}$. So the time which is cost for this movement is $0.01 \mathrm{~s}$, and the motion equation can be written as:

$$
\left.\begin{array}{l}
x(t)=x_{0}+s(t) \cos \varphi \\
y(t)=y_{0}+s(t) \sin \varphi \\
v_{x}(t)=v(t) \cos \varphi \\
v_{y}(t)=v(t) \sin \varphi
\end{array}\right\}
$$


where $x_{0}$ and $y_{0}$ represent the initial positions of the actuator (point $P$ ) along $X$ and $Y$ axis. $v_{x}$ and $v_{y}$ represent the initial velocities of the actuator along $X$ and $Y$ axis. $s(t)$ and $v(t)$ represent the displacement and velocity of the actuator, respectively.

Through calculating the elastic displacement, velocity and acceleration by the Matlab, the change rules can be obtained, which are shown in Fig. 12 and Fig. 13.

Fig. 12 (a) shows the elastic angular displacement around $Z$ axis. Fig. 12 (b) and Fig. 12 (c)

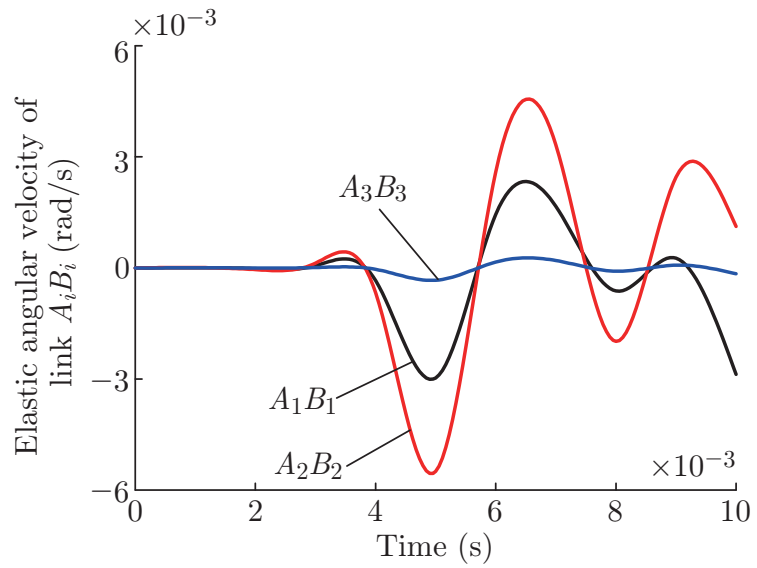

(a) Elastic angulr velocity of link $A_{i} B_{i}$

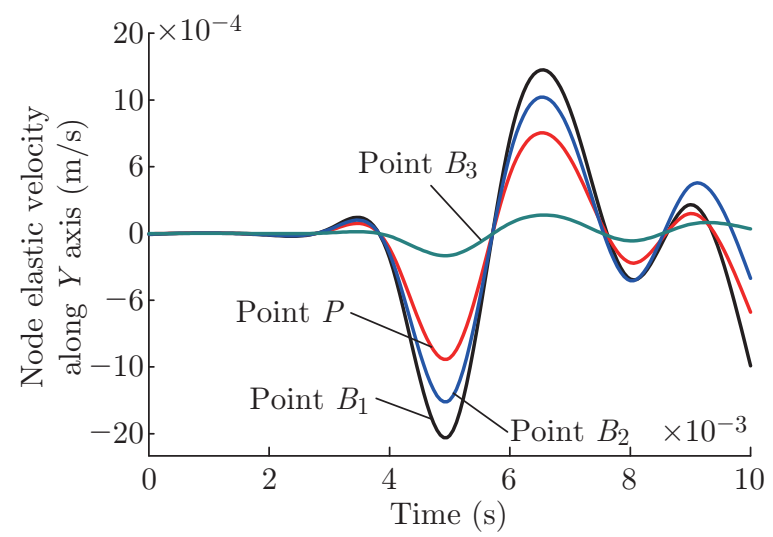

(c) Node elastic velocity along $Y$ axis

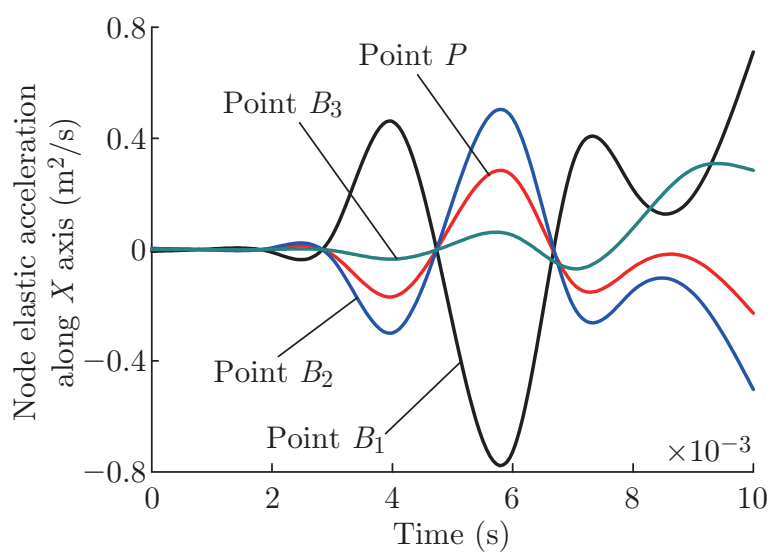

(e) Node elastic acceleration along $X$ axis

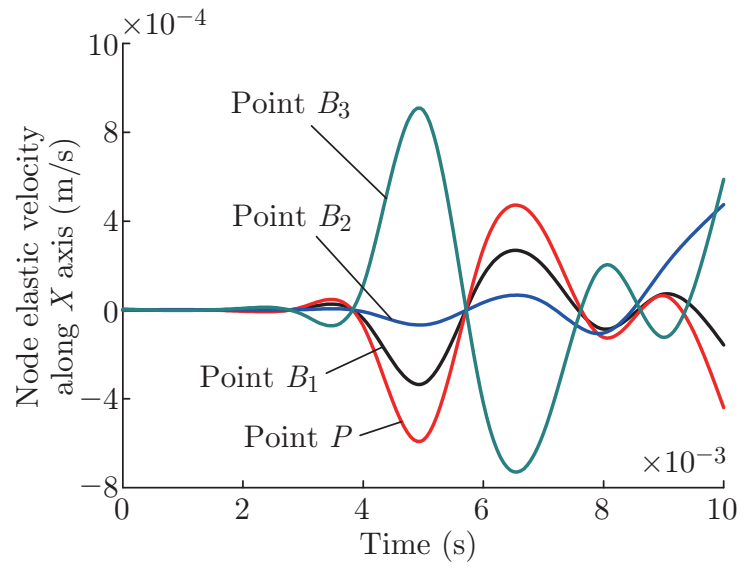

(b) Node elastic velocity along $X$ axis

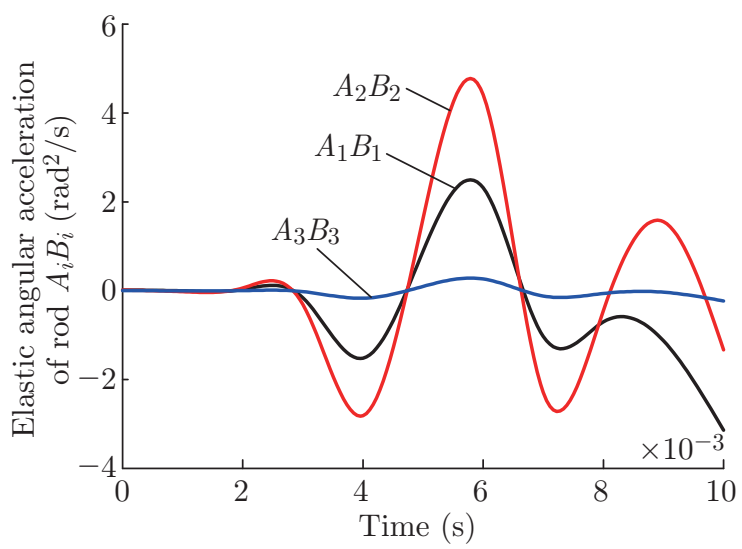

(d) Elastic angular acceleration of link $A_{i} B_{i}$

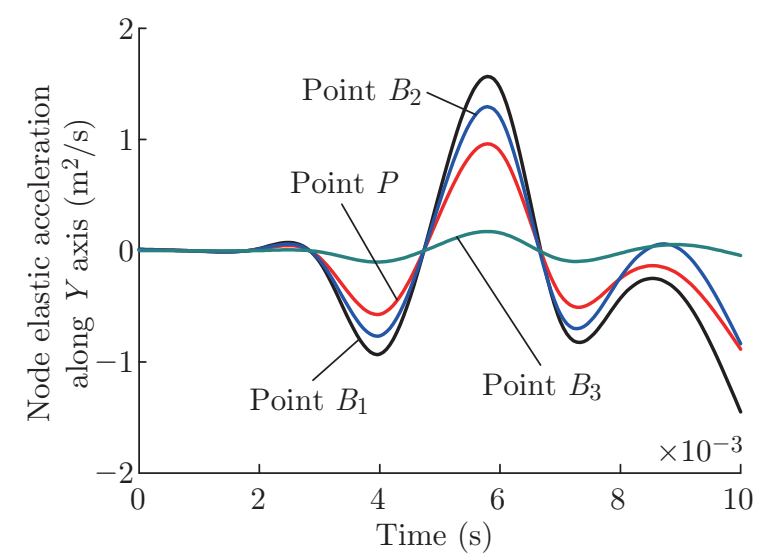

(f) Node elastic acceleration along $Y$ axis

Fig. 13: Elastic velocity and acceleration of the PPM 
show the elastic displacement along $X$ axis and $Y$ axis. By analyzing the results, it can be seen that the maximum elastic displacement caused by the elastic deformation is $0.0036 \mathrm{~mm}$ which is smaller than the permissible elastic displacement, so the PPM satisfies with the requirement of the moving stiffness. Through calculating the finite element model of the PPM, the effect of the elastic deformation on the velocity and acceleration of the terminal actuator in the PPM can be obtained and shown in Fig. 13.

Fig. 13 (c) shows that the maximum velocity vibration along $Y$ axis is $0.0015 \mathrm{~m} / \mathrm{s}$. As shown in Fig. 13 (f), the acceleration vibration along $Y$ axis is up to $1.5 \mathrm{~m} / \mathrm{s}^{2}$. Through analyzing the velocity and acceleration curves, it shows that the elastic deformations of the components in the PPM would cause the serious reciprocating vibration of the velocity and acceleration at the actuator. The vibration is greatly different in each direction and it is determined by the structural features, motion regulation and force conditions of the PPM. The moving scale along $X$ axis is much greater than that along $Y$ axis as shown in Fig. 12 (b) and Fig. 12 (c). The stiffness values of the PPM change with different positions, so the elastic displacement, velocity and acceleration are closely related to the moving status of the PPM. The moving stiffness results provide a theory base for controlling the vibration and improving the motion accuracy.

\section{Conclusion}

In this paper, the FEM is applied to study the integrated stiffness of a 3-RR planner parallel manipulator with actuation redundancy. The integrated stiffness model of the PPM is established based on a planar beam element which is able to analyze the static stiffness and the dynamic stiffness and the moving stiffness comprehensively. Three stiffness calculation and simulation results demonstrate that the PPM meets the work requirements. In addition, the moving stiffness calculation results show that the elastic velocity and elastic acceleration lead to reciprocating vibration of the PPM, so effective methods should be used to eliminate the negative effects in order to improve the motion accuracy. The integrated stiffness modeling unifies different stiffness models which improve the efficiency for the mechanism design, structure optimization and motion control, so the integrated stiffness modeling based on the FEM is worth applying for parallel manipulators.

\section{Acknowledgments}

Financial support for this work, provided by the National Natural Science Foundation of China (No. 51275512), the Priority Academic Program Development of Jiangsu Higher Education Institutions and the Fundamental Research Funds for the Central Universities (No. 2012QNA28), is gratefully acknowledged.

\section{References}

[1] K. Alexandr, C. Damien, P. Anatol, Stiffness modeling for perfect and non-perfect parallel manipulators under internal and external loadings, Mechanism and Machine Theory, 79 (2014), 1-28 
[2] F. Majou, C. Gosselin, P. Wenger, D. Chablat, Parametric stiffness analysis of the Orthoglide, Mechanism and Machine Theory, 42 (2007), 296-311

[3] A. Jokin, Z. Isidro, A. Oscar, N. Jasiel, Improving static stiffness of the image parallel manipulator using inverse singularities, Robotics and Computer-Integrated Manufacturing, 28 (2012), 458-471

[4] B. Hu, Y. Lu, Q. Tan, J. P. Yu, J. D. Han, Analysis of stiffness and elastic deformation of a 2(SP+SPR+SPU) serial-parallel manipulator, Robotics and Computer-Integrated Manufacturing, 27 (2011), 418-425

[5] G. Cheng, P. Xu, D. H. Yang, H. G. Liu, Stiffness analysis of a 3CPS parallel manipulator for mirror active adjusting platform in segmented telescope, Robotics and Computer-Integrated Manufacturing, 29 (2013), 302-311

[6] Y. M. Song, H. Gao, T. Sun, G. Dong, B. B. Lian, Y. Qi, Kinematic analysis and optimal design of a novel 1T3R parallel manipulator with an articulated travelling plate, Robotics and ComputerIntegrated Manufacturing, 30 (2014), 508-516

[7] M. Ceccarelli, G. Carbone, A stiffness analysis for CaPaMan (Cassino Parallel Robot), Mechanism and Machine Theory, 37 (2002), 427-439

[8] R. Amir, A. Alireza, M. N. Payam, A. T. Mohammad, Position, Jacobian and workspace analysis of a 3-PSP spatial parallel manipulator, Robotics and Computer-Integrated Manufacturing, 29 (2013), 158-173

[9] P. Anatol, K. Alexandr, C. Damien, Enhanced stiffness modeling of manipulators with passive joints, Mechanism and Machine Theory, 46 (2011), 662-679

[10] F. Gómez-Bravo, G. Carbone, J. C. Fortes, Collision free trajectory planning for hybrid manipulators, Mechatronics, 22 (2012), 836-851

[11] M. Ebrahim, A survey of bio-inspired robotics hands implementation: New directions in dexterous manipulation, Robotics and Autonomous Systems, 61 (2013), 517-544

[12] D. Fabio, I. Hiroshi, Non-singular transitions between assembly modes of 2-DOF planar parallel manipulators with a passive leg, Mechanism and Machine Theory, 77 (2014), 182-197

[13] J. Wu, T. M. Li, J. S. Wang, L. P. Wang, Stiffness and natural frequency of a 3-DOF parallel manipulator with consideration of additional leg candidates, Robotics and Autonomous Systems, 61 (2013), 868-875

[14] H. Giberti, S. Cinquemani, S. Ambrosetti, 5R 2DoF parallel kinematic manipulator - A multidisciplinary test case in mechatronics, Mechatronics, 23 (2013), 949-959

[15] G. Legnani, I. Fassi, H. Giberti, S. Cinquemani, D. Tosi, A new isotropic and decoupled 6-DoF parallel manipulator, Mechanism and Machine Theory, 58 (2012), 64-81

[16] A. Cammarata, R. Sinatra, Elastodynamic optimization of a 3T1R parallel manipulator, Mechanism and Machine Theory, 73 (2014), 184-196 